\title{
Sclérose tubéreuse de Bourneville
}

Parmi les phacomatoses, la sclérose tubéreuse de Bourneville (ou tuberous sclerosis complex, TSC) a été un peu négligée par les généticiens ; comme les autres phacomatoses, la maladie se transmet selon le mode autosomique dominant ; les travaux de génétique moléculaire portant sur TSC sont bien plus rares que ceux concernant la neurofibromatose de Von Recklinghausen ou la maladie de Von Hippel-Lindau (il suffit de lire $\mathrm{m} / \mathrm{s}$ pour s'en convaincre...).

La sclérose tubéreuse est caractérisée par l'atteinte de multiples tissus; seuls les muscles striés, les nerfs périphériques, les méninges, le thymus et la glande pinéale sont épargnés. Les organes le plus souvent atteints sont la peau (adénomes sébacés ou angiofibromes de la face, macules hypopigmentées, fibromes unguéaux ou tumeurs de Koenen), le système nerveux central, le rein, le cœur et le poumon (voir plus loin). L'incidence de la maladie à la naissance est d'environ 1 sur 10000 . La prévalence dans la population adulte est d'environ 1 sur 30000 ; cela suggère que l'espérance de vie des malades est moindre que celle de la population générale. En outre, la prévalence dans les établissements pour enfants handicapés est d'environ 1 sur 300 . En effet, le retard mental est une des conséquences les plus graves de TSC ; 50 à $60 \%$ des malades atteints ont un retard mental et ceuxci ont presque tous une comitialité associée. Cependant, l'expression clinique de la maladie est très variable d'une famille à l'autre et au sein d'une même famille.

L'étude faite à la Mayo Clinic (Rochester, USA) porte sur la mortalité dans cette maladie [1]. Parmi 355 malades suivis dans cet hôpital, 40 sont décédés d'une cause directement imputable à TSC. Les causes cardiovasculaires (tumeurs bénignes mes et anévrismes de l'aorte thoracique) prédominent dans l'enfance. Parmi 91 malades étudiés par échographie cardiaque, 45 (49\%) avaient un rhabdomyome. Les complications rénales représentent la cause la plus fréquente de la mort à partir de l'âge de 10 ans et surtout chez l'adulte : sept malades sont décédés d'insuffisance rénale (souvent liée au développement de kystes multiples), deux d'une hémorragie due à un angiomyolipome rénal (tumeur vasculaire et graisseuse bénigne, fréquente dans TSC) et deux de carcinome rénal (cette association a déjà été signalée et rappelle ce qu'on observe dans la maladie de Von HippelLindau ( $m / s n^{\circ} 6$, vol. 7, p. 632). Dix malades sont décédés des conséquences d'une tumeur cérébrale (astrocytome à cellules géantes). Il faut signaler également que, chez 13 malades ayant une débilité mentale grave due à TSC, un état de mal épileptique ou une bronchopneumopathie a été à l'origine de la mort. Enfin une lymphangiomyomatose pulmonaire a été démontrée chez 10 malades, responsable de la mort chez quatre d'entre eux, tous âgés de 40 ans ou plus [1].

Dans l'éditorial qui accompagne cet article [2], Stefansson fait le point sur quelques aspects de la pathogénie. L'atteinte neurologique pourrait résulter d'une anomalie de migration et d'orientation des astrocytes et des neurones. Des tissus tumoraux (angiofibromes, angiomyolipomes ou astrocytomes) en culture produisent des cellules particulières " monstrueuses " dont le type et l'origine restent inconnus.

La maladie est transmise de façon autosomique dominante mais des néomutations pourraient être impliquées dans près de $60 \%$ des cas. Dans $50 \%$ des familles étudiées, on observe une liaison à des marqueurs (groupes sanguins $\mathrm{ABO}$ et oncogène
Abelson) localisés à l'extrémité télomérique du bras long du chromosome 9 (9p34) (locus TSC1) [3]. Un second locus, TSC2, a été identifié dans d'autres familles et localisé au chromosome 11 (11q 22-23). L'analyse faite par Janssen et al. [4] suggère que les produits des deux gènes, situés aux deux loci différents, seraient nécessaires au développement du phénotype normal [3]. Certains gènes candidats ont déjà été étudiés : le gène bexabrachion (ténascine) qui est localisé en 9p34 et code pour une protéine impliquée dans l'adhérence et la prolifération cellulaires, a pu être exclu car il est de localisation plus centromérique que le locus TSC. Un autre gène candidat, celui de la prostaglandine $\mathrm{D}_{2}$ synthétase, localisé en 9q34.2, est en cours d'étude par Stefansson. Le gène du récepteur dopaminergique $\mathrm{D}_{2}$ représente un gène candidat pour TSC2 [4].

Il reste encore beaucoup à faire pour identifier le ou les défauts moléculaires en cause dans la sclérose tubéreuse. Il est bon de rappeler quelques règles cliniques simples concernant la surveillance de ces malades : l'échographie cardiaque et rénale, la radiographie du thorax et l'imagerie du cerveau par résonance magnétique sont indispensables de façon périodique pour détecter à temps certaines localisations curables de la sclérose tubéreuse.

J.P.G.

1. Shepherd CW, Gomez MR, Lie JT, et al Causes of death in patients with tuberous sclerosis. Mayo Clin Proc 1991; 66 : 792-6. 2. Stefansson M. Tuberous sclerosis. Mayo Clin Proc 1991; 66 : 868-72.

3. Haines JL, Short MP, Kwiatkowski DJ, et al. Localization of one gene for tuberous sclerosis within $9 \mathrm{q} 32-9 \mathrm{q} 34$ and further evidence for heterogeneity. Am Hum Genet 1991; 49 : 764-72.

4. Janssen LAJ, Standkuyl LA, Merkens EC, et al. Genetic heterogeneity in tuberous sclerosis. Genomics $1990 ; 8: 237-49$. 